\title{
On an inductive construction of higher spin Dirac operators
}

\author{
H. De Schepper*, D. Eelbode ${ }^{\dagger}$ and T. Raeymaekers* \\ *Clifford research group, Department of Mathematical Analysis, Ghent University \\ Galglaan 2, 9000 Ghent, Belgium \\ ${ }^{\dagger}$ Department of Mathematics and Computer Science, University of Antwerp \\ Campus Middelheim, G-Building, Middelheimlaan 1, 2020 Antwerpen, Belgium
}

\begin{abstract}
In this contribution, we introduce higher spin Dirac operators, i.e. a specific class of differential operators in Clifford analysis of several vector variables, motivated by equations from theoretical physics. In particular, the higher spin Dirac operator in three vector variables will be explicitly constructed, starting from a description of the so-called twisted Rarita-Schwinger operator.
\end{abstract}

Keywords: Higher spin Dirac operator, Dirac operator, Rarita Schwinger operator

PACS: PACS numbers: 2.20Qs, 2.30Px

\section{INTRODUCTION}

One of the most important equations in theoretical physics is the well-known Dirac equation, see [8], describing the wave equation of the electron, which is an elementary particle characterized by a quantum spin number $s=\frac{1}{2}$. More exotic particles, such as e.g. the theoretical gravitino, are also characterized by a certain quantum spin number. The gravitino predicted in supergravity theory for example, has $s=\frac{3}{2}$. Higher half-spin particles $\left(s=\frac{5}{2}, s=\frac{7}{2}, \ldots\right)$, have been predicted as well. Note that next to these fermions with half-integer valued spin, bosonic particles with integer valued spin have been studied as well, for instance the photon with $s=1$ and the graviton with $s=2$.

The Dirac equation, governed by an elliptic first order differential operator called the Dirac operator, is also studied in Clifford analysis, however from a function theoretical point of view. This has lead to a rich higher dimensional function theory in Euclidean space, which at the same time generalizes the theory of holomorphic functions in the complex plane and refines classical harmonic analysis. One of the most important features of this function theory is the fact that it treats the dimension of the considered vector space as a parameter, which is important from a physical point of view as well, since it allows to export the obtained results to more general contexts than classical 4-dimensional spacetime, as considered for instance in string theory. Flat space string theories are 26-dimensional in the bosonic case, while superstring and $\mathrm{M}$-theories turn out to involve 10 or 11 dimensions for flat solutions. In such contexts, however, the single quantum spin number from the classical setting has to be replaced by a vector of quantum spin numbers, the so-called highest weight of the underlying symmetry group. For instance: the counterpart of the electron with spin $s=\frac{1}{2}$ in $m$-dimensional space will be represented by the highest weight vector $\left(\frac{1}{2}, \frac{1}{2}, \ldots, \frac{1}{2}\right)$, while the counterpart of the gravitino will be represented by the highest weight vector $\left(\frac{3}{2}, \frac{1}{2}, \ldots, \frac{1}{2}\right)$.

The main aim of this contribution is to consider the case of 'particles' in $m$-dimensional space represented by the highest weight vector $\left(k+\frac{1}{2}, \ell+\frac{1}{2}, \frac{1}{2}, \ldots, \frac{1}{2}\right)$ of length $\left\lfloor\frac{m}{2}\right\rfloor$. Obviously, this implies that a generalization of the classical Dirac equation will have to be considered, and that the first task will be to establish explicitly the form of the underlying operator, called a higher spin Dirac operator, governing these equations in the context of Clifford analysis in several, here three, vector variables.

A first generalization, which has been extensively studied by Rarita and Schwinger, see [10], concerns the case of the abovementioned particles with highest weight vector $\left(\frac{3}{2}, \frac{1}{2}, \ldots, \frac{1}{2}\right)$. More in general, the operator $\mathscr{R}_{k}$ corresponding to the weight vector $\left(k+\frac{1}{2}, \frac{1}{2}, \ldots, \frac{1}{2}\right)$, in a two vector variables setting, is called the Rarita-Schwinger operator and will be briefly discussed in the next section as an illustrative example. A twisted version of this Rarita-Schwinger operator will then be used as the starting point for obtaining, by means of a constructive method, an explicit expression for the higher spin Dirac operator $\mathscr{Q}_{k, \ell}$ corresponding to 'particles' with highest weight vector $\left(k+\frac{1}{2}, \ell+\frac{1}{2}, \frac{1}{2}, \ldots, \frac{1}{2}\right)$. This strategy not only leads to a direct confirmation of the results already obtained in [2,9], but moreover provides us with an inductive method for the construction of other higher spin Dirac operators as well. Furthermore, it has the advantage of clearing the way for a complete description of the set of solutions of general higher spin Dirac operators in future. 


\section{CONSTRUCTION OF THE RARITA-SCHWINGER OPERATOR}

Define $\mathbb{R}_{m}$ to be the real Clifford algebra generated by an orthonormal basis $\left(\underline{e}_{1}, \ldots, \underline{e}_{m}\right)$ of the $m$-dimensional vector space $\mathbb{R}^{m}$ and let $\mathbb{C}_{m}=\mathbb{C} \otimes \mathbb{R}_{m}$ be its complexification. The multiplication in these algebras is governed by the relations

$$
\underline{e}_{i} \underline{e}_{j}+\underline{e}_{j} \underline{e}_{i}=-2 \delta_{i j}, \quad i, j=1, \ldots, m
$$

The spinor space $\mathbb{S}$ then is defined as a minimal left ideal in $\mathbb{C}_{m}$. We refer to e.g. [1] for more background information. It has been shown (see e.g. $[11,6,7])$ that $\mathbb{S}$ is an irreducible representation of the spin group

$$
\operatorname{Spin}(m)=\left\{s=\prod_{j=1}^{2 k} s_{j}: k \in \mathbb{N}, s_{j} \in S^{m-1}\right\}
$$

with highest weight $\left(\frac{1}{2}, \frac{1}{2}, \ldots, \frac{1}{2}\right)$, which will in fact play the role of the $m$-dimensional counterpart of the electron.

Now consider one of the fundamental building blocks of Clifford analysis: the first order elliptic, Clifford vector valued, differential operator $\underline{\partial}_{x}=\sum_{j=1}^{m} \underline{e}_{j} \partial_{x_{j}}$, called the Dirac operator. This Dirac operator shows a strong connection to the irreducible representation $\mathbb{S}$ of $\operatorname{Spin}(m)$. Indeed, observe that for each spinor valued function $f(\underline{x})$ in $\mathscr{C}^{\infty}\left(\mathbb{R}^{m}, \mathbb{S}\right)$ it holds that $\partial_{x} f$ again belongs to $\mathscr{C}^{\infty}\left(\mathbb{R}^{m}, \mathbb{S}\right)$, whence this space is invariant under the Dirac operator.

The main aim behind higher spin Clifford analysis is to define and construct a Dirac-like operator $\mathscr{Q}_{\lambda}$ for each irreducible representation $\mathbb{V}_{\lambda}$ of the group $\operatorname{Spin}(m)$, with highest weight vector $\lambda$. In other words, we aim at constructing a first order differential operator which leaves the space $\mathscr{C}^{\infty}\left(\mathbb{R}^{m}, \mathbb{V}_{\lambda}\right)$ invariant.

To this end, let us first take a closer look at the fermionic particle with spin $k+\frac{1}{2}$, where $k$ is a natural number. The $m$-dimensional counterpart of this particle is the irreducible representation of $\operatorname{Spin}(m)$ with highest weight vector $\left(k+\frac{1}{2}, \frac{1}{2}, \ldots, \frac{1}{2}\right)$. It has been shown, see [6], that this representation can actually be characterized by the space $\mathscr{M}_{k}$ of $\mathbb{S}$ valued polynomials $P(\underline{u})$ of homogeneity degree $k$ in the vector variable $\underline{u}$, for which $\underline{\partial}_{u} P=0-$ we call these polynomials monogenic. So, similarly to the behaviour of the standard Dirac operator, we now want to find a first order differential operator $\mathscr{R}_{k}$, acting on functions $f(\underline{x}, \underline{u})$ from the space $\mathscr{C}^{\infty}\left(\mathbb{R}^{m}, \mathscr{M}_{k}\right)$, and leaving this space invariant, i.e.

$$
\mathscr{R}_{k}: \mathscr{C}^{\infty}\left(\mathbb{R}^{m}, \mathscr{M}_{k}\right) \rightarrow \mathscr{C}^{\infty}\left(\mathbb{R}^{m}, \mathscr{M}_{k}\right)
$$

To fix the ideas, we will briefly describe how this operator is constructed. To this end, first let $\mathscr{H}_{k}$ be the usual space of complex valued harmonic polynomials $P(\underline{u})$ of homogeneity degree $k$ in the vector variable $\underline{u}$, and consider $\mathscr{H}_{k} \otimes \mathbb{S}$, i.e., allow the polynomials to take their values in $\mathbb{S}$. We then introduce the so-called twisted Dirac operator, given by

$$
\underline{\partial}_{x}^{T}=\mathbf{1}_{u} \otimes \underline{\partial}_{x}: \mathscr{C}^{\infty}\left(\mathbb{R}^{m}, \mathscr{H}_{k} \otimes \mathbb{S}\right) \rightarrow \mathscr{C}^{\infty}\left(\mathbb{R}^{m}, \mathscr{H}_{k} \otimes \mathbb{S}\right)
$$

Observe indeed that the action of the Dirac operator in the vector variable $\underline{x}$ will not affect the fact that the values taken are harmonic in the vector variable $\underline{u}$. Now, since the Laplacian $\Delta_{u}$ is factorized by the Dirac operator $\underline{\partial}_{u}$, i.e. $\Delta_{u}=-\underline{\partial}_{u}^{2}$, it is clear that $\mathscr{M}_{k} \subset \mathscr{H}_{k} \otimes \mathbb{S}$. Moreover, see [3], $\mathscr{H}_{k} \otimes \mathbb{S}$ decomposes as the direct sum $\mathscr{M}_{k} \oplus \underline{u} \mathscr{M}_{k-1}$, thus containing a unique copy of $\mathscr{M}_{k}$. Hence, in order to arrive at the desired Rarita-Schwinger operator $\mathscr{R}_{k}$, one only needs to effectuate the projection from $\mathscr{H}_{k} \otimes \mathbb{S}$ onto its component $\mathscr{M}_{k}$. More precisely, one needs to find a projection operator $\pi_{k}$ such that for all $f(\underline{x}, \underline{u})$ in $\mathscr{C}^{\infty}\left(\mathbb{R}^{m}, \mathscr{M}_{k}\right)$, it holds that $\pi_{k} \underline{\partial}_{x}^{T} f(\underline{x}, \underline{u}) \in \mathscr{C}^{\infty}\left(\mathbb{R}^{m}, \mathscr{M}_{k}\right)$. Direct calculations then reveal the explicit form of the Rarita-Schwinger operator to be

$$
\mathscr{R}_{k}=\left(1+\frac{\underline{u} \underline{\partial}_{u}}{m+2 k-2}\right) \underline{\partial}_{x}
$$

see also $[4,5]$.

Remark 1 In fact, on account of the direct sum decomposition $\mathscr{H}_{k} \otimes \mathbb{S}=\mathscr{M}_{k} \oplus \underline{u} \mathscr{M}_{k-1}$, the twisted Dirac operator decomposes into two operators. These are the natural operators acting on the considered functions: the RaritaSchwinger operator $\mathscr{R}_{k}$ and a so-called twistor operator originating from the projection onto the space $\mathscr{M}_{k-1}$, usually denoted as

$$
\mathscr{T}_{k-1}^{k}: \mathscr{C}^{\infty}\left(\mathbb{R}^{m}, \mathscr{M}_{k}\right) \rightarrow \mathscr{C}^{\infty}\left(\mathbb{R}^{m}, \mathscr{M}_{k-1}\right)
$$

For further details, we again refer to [4, 5]. 


\section{THE TWISTED RARITA-SCHWINGER OPERATOR}

In the previous section, we have used the definition of the Dirac operator (in one vector variable) for the construction of the Rarita-Schwinger operator (in two vector variables). The philosophy behind this approach is that a generalization of it will lead to an inductive principle for the explicit construction of the higher spin Dirac operator in $n$ variables, starting from the twisted higher spin Dirac operator in $n-1$ variables.

In the remainder of this contribution, we will give the explicit results for the construction of the higher spin Dirac operator $\mathscr{Q}_{k, l}$ in three vector variables. Following the general reasoning, this operator will have to correspond to the irreducible representation of the group $\operatorname{Spin}(m)$ with highest weight vector $\left(k+\frac{1}{2}, \ell+\frac{1}{2}, \frac{1}{2}, \ldots, \frac{1}{2}\right)$. It has been proven that this is in fact the space $\mathscr{S}_{k, \ell}$ of $\mathbb{S}$ valued polynomials $f(\underline{u}, \underline{v})$ with bidegree of homogeneity $(k, \ell)$ in the vector variables $(\underline{u}, \underline{v})$, for which it moreover holds that $\underline{\partial}_{u} f=\underline{\partial}_{v} f=\left\langle\underline{u}, \underline{\partial}_{v}\right\rangle f=0$. We call these polynomials simplicial monogenic. Thus, we are looking for a first order differential operator leaving the space $\mathscr{C}^{\infty}\left(\mathbb{R}^{m}, \mathscr{S}_{k, \ell}\right)$ invariant, i.e.

$$
\mathscr{Q}_{k, \ell}: \mathscr{C}^{\infty}\left(\mathbb{R}^{m}, \mathscr{S}_{k, \ell}\right) \rightarrow \mathscr{C}^{\infty}\left(\mathbb{R}^{m}, \mathscr{S}_{k, \ell}\right)
$$

In order to determine the explicit form of this operator, we define the twisted Rarita-Schwinger operator as follows:

$$
\mathscr{R}_{k}^{T}=\mathbf{1}_{v} \otimes \mathscr{R}_{k}: \mathscr{C}^{\infty}\left(\mathbb{R}^{m}, \mathscr{H}_{\ell} \otimes \mathscr{M}_{k}\right) \rightarrow \mathscr{C}^{\infty}\left(\mathbb{R}^{m}, \mathscr{H}_{\ell} \otimes \mathscr{M}_{k}\right)
$$

Since clearly, $\mathscr{S}_{k, \ell}$ is an irreducible submodule of $\mathscr{H}_{\ell} \otimes \mathscr{M}_{k}$, we are able to follow a similar strategy as above: to determine $\mathscr{Q}_{k, \ell}$ by application of the twisted Rarita-Schwinger operator on the space $\mathscr{C}^{\infty}\left(\mathbb{R}^{m}, \mathscr{S}_{k, \ell}\right)$ and subsequent projection on the same space. Now, the following decomposition of the action of the twisted Rarita-Schwinger operator can be obtained.

Proposition 1 For all $k \geqslant \ell$, there exists a constant $\gamma_{k, \ell} \in \mathbb{R}$ such that

$$
\mathscr{R}_{k}^{T} f=\phi_{0}+\gamma_{k, \ell} \pi_{k}[\underline{v}]\left\langle\underline{\partial}_{v}, \underline{\partial}_{x}\right\rangle f, \quad \forall f \in \mathscr{C}^{\infty}\left(\mathbb{R}^{m}, \mathscr{S}_{k, \ell}\right)
$$

where the component $\phi_{0}$ satisfies the conditions $\underline{\partial}_{u} \phi_{0}=\underline{\partial}_{v} \phi_{0}=\left\langle\underline{u}, \underline{\partial}_{v}\right\rangle \phi_{0}=0$, and $\pi_{k}[\underline{v}]$ is defined as

$$
\pi_{k}[\underline{v}]=\left(1+\frac{\underline{u} \underline{\partial}_{u}}{m+2 k-2}\right) \underline{v}
$$

Moreover, it holds that $\phi_{0} \in \mathscr{C}^{\infty}\left(\mathbb{R}^{m}, \mathscr{S}_{k, \ell}\right)$ and $\left\langle\underline{\partial}_{v}, \underline{\partial}_{x}\right\rangle f \in \mathscr{C}^{\infty}\left(\mathbb{R}^{m}, \mathscr{S}_{k, \ell-1}\right)$.

In order to prove the uniqueness of this decomposition, it remains to show that $\mathscr{S}_{k, \ell} \oplus \mathscr{S}_{k, \ell-1}$ is contained in $\mathscr{H}_{\ell} \otimes \mathscr{M}_{k}$ with multiplicity one. To this end, observe that $\mathscr{H}_{\ell} \otimes \mathscr{M}_{k} \subset \mathscr{H}_{k} \otimes \mathscr{H}_{\ell} \otimes \mathbb{S}$ and consider the following decomposition of the latter space, see [9].

Lemma 1 For all $k \geq \ell \geq 0$, the tensor product $\mathscr{H}_{k} \otimes \mathscr{H}_{\ell} \otimes \mathbb{S}$ decomposes as the direct sum

$$
\mathscr{H}_{k} \otimes \mathscr{H}_{\ell} \otimes \mathbb{S}=\bigoplus_{i=0}^{\ell} \bigoplus_{j=0}^{i} \mathscr{H}_{k+\ell-2 i+j, j} \otimes \mathbb{S}
$$

Taking into consideration that

$$
\mathscr{H}_{k, \ell} \otimes \mathbb{S} \cong \mathscr{S}_{k, \ell} \oplus\left(1-\delta_{\ell, 0}\right) \mathscr{S}_{k, \ell-1} \oplus\left(1-\delta_{k, \ell}\right) \mathscr{S}_{k-1, \ell} \oplus\left(1-\delta_{\ell, 0}\right) \mathscr{S}_{k-1, \ell-1}
$$

one can calculate that $\mathscr{S}_{k, \ell}$ is contained in $\mathscr{H}_{k} \otimes \mathscr{H}_{\ell} \otimes \mathbb{S}$ with multiplicity one, and $\mathscr{S}_{k, \ell-1}$ with multiplicity two. However, taking restrictions to $\mathscr{H}_{\ell} \otimes \mathscr{M}_{k}$, both components $\mathscr{S}_{k, \ell}$ and $\mathscr{S}_{k, \ell-1}$ are found to appear with multiplicity one, which can also be seen by noticing that $\left(\mathscr{H}_{\ell} \otimes \mathscr{H}_{k}\right)=\mathscr{H}_{\ell} \otimes \mathscr{M}_{k} \oplus\left(\mathscr{H}_{\ell} \otimes \mathscr{M}_{k-1}\right)$.

Returning now to the construction of $\mathscr{Q}_{k, \ell}$, it only remains to explicitly obtain the component $\phi_{0}$.

Theorem 1 In the decomposition of the twisted Rarita-Schwinger operator the component $\phi_{0} \in \mathscr{C}^{\infty}\left(\mathbb{R}^{m}, \mathscr{S}_{k, \ell}\right)$, defined in Proposition 1, is explicitly given by

$$
\phi_{0}=\left(1+\frac{\underline{u} \underline{\partial}_{u}}{m+2 k-2}\right)\left(1+\frac{\underline{v} \underline{\partial}_{v}}{m+2 \ell-4}\right) \underline{\partial}_{x} f
$$

This means that the higher spin Dirac operator $\mathscr{Q}_{k, \ell}$ takes the form

$$
\mathscr{Q}_{k, \ell}=\left(1+\frac{\underline{u} \underline{\partial}_{u}}{m+2 k-2}\right)\left(1+\frac{\underline{v} \underline{\partial}_{v}}{m+2 \ell-4}\right) \underline{\partial}_{x}
$$


Observe that this result agrees with the operator obtained in [9] by means of another approach.

This inductive method for the construction of the higher spin Dirac operator in $n$ vector variables, based on the twisted higher spin Dirac operator in $n-1$ vector variables, turns out to work properly in the general case as well, as we will show in a forthcoming paper.

\section{REFERENCES}

1. Brackx, F., Delanghe, R., Sommen, F., Clifford Analysis, Research Notes in Mathematics 76, Pitman, London, 1982.

2. Brackx, F., Eelbode, D., Van de Voorde, L., Polynomial solutions for higher spin Dirac operators in three vector variables, submitted.

3. Brackx, F., Constales, D., Ronveaux, A., Serras, H., On the Harmonic and Monogenic Decomposition of Polynomials, $J$. Symbol. Comput. 8 (1989), pp. 297-304

4. Bureš, J., Sommen, F., Souček, V., Van Lancker, P., Rarita-Schwinger type operators in Clifford analysis, J. Funct. Anal. 185, pp. 425-456.

5. Bureš, J., Sommen, F., Souček, V., Van Lancker, P., Symmetric analogues of Rarita-Schwinger equations, Ann. Glob. Anal. Geom. 21(no.3) (2001), pp. 215-240.

6. Constales, D., Sommen. F., Van Lancker, P., Models for irreducible representations of Spin $(m)$, Adv. Appl. Cliff. Alg. 11(S1) (2001), pp. 271-289.

7. Delanghe, R., Sommen, F., Souček, V., Clifford analysis and spinor valued functions, Kluwer Academic Publishers, Dordrecht, 1992.

8. Dirac, P.A.M, The quantum theory of the electron, Proceedings of the Royal Society of London 117, 1928

9. Eelbode, D., Van de Voorde, L., A toy model for higher spin Dirac operators, Physics of Atomic Nuclei 73(2) (2010) pp. 282-287.

10. Rarita, W., Schwinger, J., On a theory of particles with half-integer spin, Phys. Rev. 60, 61 - 61, 1941

11. Stein, E.W. , Weiss, G., Generalization of the Cauchy-Riemann equations and representations of the rotation group, Amer. J. Math. 90 (1968), pp. 163-196. 